\title{
La educación transmedia como estrategia innovadora para integrar lengua y cultura en la enseñanza de ELE
}

\author{
Transmedia education as an innovative strategy for integrating \\ language and culture in the teaching of Spanish as a Foreign \\ Language
}

\author{
CARMEn HerRero \\ Manchester Metropolitan University and Film in language teaching \\ ASSOCIATION \\ c.herrero@mmu.ac.uk \\ https://orcid.org/0000-0002-1392-4224
}

\begin{abstract}
Resumen
Este artículo recoge diversas experiencias didácticas encaminadas a integrar el aprendizaje de lengua y cultura. El objetivo es plantear cómo se puede desarrollar una sinergia entre las clases de español como lengua extranjera (L2) y las asignaturas de contenidos mediante diversas estrategias de comunicación transmedia. En la primera parte se examinan diversos modelos pedagógicos en el contexto de enseñanza de L2 en el Reino Unido. En la segunda, presentamos los proyectos de transmediaa en L2 que se están realizando desde el Grupo de Investigación Film, Languages and Media in Education (FLAME). El último apartado incluye las conclusiones y futuras líneas de investigación.

Palabras clave: Aprendizaje por contenido; Aprendizaje integrado de Contenidos y Lenguas Extranjeras (AICLE); experiencias didácticas; ELE; transmedia.
\end{abstract}

\footnotetext{
Abstract

This article collects several didactic experiences aimed at integrating the learning of language and culture. The objective is to propose how a synergy can be developed between $\mathrm{L} 2$ classes and content subjects through transmedia narrative strategies. The first part examines different pedagogical models in the content of $L 2$ teaching in the United Kingdom. The second presents the projects on transmedia and L2 carried out in this area by the Research Group Film, Languages and Media in Education of Manchester Metropolitan University (FLAME). The last section includes the conclusions and future lines of research are proposed.
} 
Keywords: Content-based instruction; Content and Language Integrated Learning (CLIL); didactic experiences; ELE; transmedia 


\section{Introducción}

El panorama del aprendizaje de lenguas en Gran Bretaña está dominado por una crisis que ha ido reduciendo paulatinamente el número de estudiantes de lenguas extranjeras (L2) tanto en secundaria como a nivel universitario (British Academy, 2019; Bowler, 2020; Parris, 2020). En el caso de Inglaterra, el hecho de que desde el 2004 la enseñanza de idiomas fuera una asignatura optativa a partir del inicio de los estudios de Key Stage 4 (estudiantes de 14 años) condujo a una merma de estudiantes que optaban por aprender una L2 en la educación secundaria post-obligatoria (GCSE y A-level). Este descenso ha afectado sobre todo a francés y alemán, mientras que las cifras para español han sido relativamente mejores. Para compensar esta situación el Ministerio de Educación británico (Departament for Education) introdujo varias medidas encaminadas a apoyar el estudio de lenguas extranjeras. En primer lugar, en 2011 se lanza el denominado English Baccalaureate (EBacc) que proponía como unos de los objetivos principales incrementar el número de estudiantes de Secundaria Obligatoria que optaban por continuar con una L2. El EBacc tenía en cuenta los resultados obtenidos en los centros educativos a partir del porcentaje de aprobados en GCSE en una lengua extranjera y otras asignaturas (inglés, matemáticas, ciencias, historia o geografía). Otra de las medidas para contrarrestar esta contracción fue la inclusión de lenguas extranjeras como parte del currículo de Primaria en 2014. Asimismo, es importante señalar que la política educativa en Escocia ha apoyado el aprendizaje de una lengua extranjera desde el primer curso de Primaria, y una segunda lengua desde el quinto curso de Primaria. ${ }^{1}$ Recientemente, el New Curriculum for Languages de Gales pretende potenciar el aprendizaje del galés, inglés y otros idiomas aplicando una estrategia multilingüe y plurilingüe (Gorrara et al., 2020).

No obstante, otras circunstancias han contribuido a la significativa disminución en el número de estudiantes que continúan con el estudio de una lengua extranjera en Secundaria y a nivel universitario. En primer lugar, la decisión del Reino Unido de abandonar la Unión Europea tras el referéndum del 2016, conocido como Brexit, ha impulsado una tendencia hacia el monolingüismo sustentada en la idea de que el inglés como lengua franca resulta suficiente para la comunicación a nivel internacional (Comisión Europea, 2019; Broady, 2020; Kelly, 2020). A lo largo de la última década se ha evidenciado el déficit lingüístico del Reino Unido que debe ser superado si desea convertirse en un país conectado globalmente. Para contrarrestar esta situación, diversas instituciones han reclamado una política que impulse la educación de enseñanza de lenguas desde primaria hasta nivel universitario y posgrado (British Academy, 2016; British Academy, 2019). En segundo lugar, la motivación para continuar con el aprendizaje de lenguas ha sido afectada por la tendencia a calificar de forma más severa los exámenes de segundas lenguas, lo que repercute a la nota global que obtienen los estudiantes y que se tiene en cuenta para ser

\footnotetext{
${ }^{1}$ https://www.gov.scot/publications/language-learning-scotland-12-approach/
} 
admitido en la universidad. Otro de los factores que supone un hándicap es la escasez de profesorado formado para la enseñanza de idiomas, particularmente en el caso de la Educación Primaria (Denechi et al., 2020). El Brexit ha agudizado también esta situación con una drástica reducción de candidatos matriculados para la obtención del Certificado de Posgrado en Educación (PGCE). Esto se debe en buena medida a que estos programas se nutrían de licenciados europeos que ahora no pueden acceder a las becas otorgadas por el gobierno para incrementar el número de docentes por medio de este Máster en Formación del Profesorado de Educación en Primaria y Secundaria. Finalmente, la excepcional situación en la que vivimos debido al impacto del Covid-19 ha afectado de forma negativa a la enseñanza de lenguas en Primaria y Secundaria: la reducción o suspensión de la enseñanza de lenguas, la falta de actividades internacionales en los colegios y una reducción en el número de estudiantes que se matricularon en GCSE y A Level para lenguas modernas (Collen, 2021). Este impacto ha sido todavía más significativo en las escuelas públicas donde, entre otros factores, la ausencia de auxiliares de conversación ha incrementado la brecha social y educativa en la provisión de lenguas extranjeras.

Como es lógico el descenso en el número de estudiantes de lenguas a nivel secundario ha influido directamente en las universidades británicas (Worton, 2009). Como consecuencia de este decrecimiento progresivo de candidatos universitarios que optan por una licenciatura de lenguas modernas, desde el año 2000 más de 50 departamentos de lenguas de diversas instituciones universitarias británicas han cerrado (Kelly, 2018). Por otra parte, los intercambios de estudiantes con instituciones universitarias europeas a través de Erasmus + han tenido que ser revisados, lo que impacta de manera directa a las licenciaturas de lenguas modernas que incluyen entre 5 y 9 meses de estancia en uno de los países de la lengua que se estudia. En esta etapa post-Brexit, un nuevo programa (Turing Scheme) ha sido implementado por primera vez para el año académico 2021-22, aunque es temprano para analizar su impacto y el futuro del mismo. Si bien es cierto que este plan ofrece nuevas oportunidades para que los estudiantes universitarios realicen su estancia internacional fuera de la Unión Europea (por ejemplo, con los países de Latinoamérica), los requisitos y restricciones para los viajes internacionales debido al COVID-19 ha perjudicado considerablemente la movilidad de los estudiantes universitarios desde el 2020.

Todas estas circunstancias han acentuado la crisis en la que se encuentran los departamentos de lenguas del Reino Unido desde hace más de una década. Uno de los factores que está contribuyendo a la agudización de esta crisis es la tendencia a presentar la enseñanza de lengua y cultura como entidades separadas, hecho que modifica la percepción del aprendiz de estos dos conceptos (Gieve y Cunico, 2012; Parks, 2020a; 2020b). En muchas ocasiones, esta distinción se agudiza con el modo en que dan esas clases y el perfil del profesorado. Las asignaturas de "contenido" (cultura, historia, cine, literatura, traducción, etc.) se suelen impartir en inglés por 
profesores que son especialistas en estas áreas, pero no necesariamente en la enseñanza de L2. También es cierto que, en reiteradas ocasiones, la elección de la lengua inglesa se debe a razones económicas, ya que permite ofrecer estas asignaturas a alumnos que no estudian una L2 y aumentar el número de inscritos en dichas unidades. Por otra parte, los profesores de lenguas son vistos como expertos en la enseñanza de una determinada lengua y su docencia se realiza en la lengua meta. Esta separación profesional acentúa una disociación que, en ocasiones, va unida a la adscripción a departamentos diferentes (Centro de Idiomas / Departamento). Todo ello hace que la colaboración entre docentes de contenidos y profesores de lengua sea esencial (Zappa-Hollman, 2018; Wilkinson, 2020).

Partiendo del contexto que acabamos de describir, el objetivo del presente trabajo es plantear cómo se puede fomentar una sinergia entre las clases de español como lengua extranjera (ELE) y las asignaturas de contenidos mediante diversas estrategias de comunicación transmedia. Basándonos en las experiencias curriculares y extracurriculares llevadas a cabo en Manchester Metropolitan University, este artículo examina de qué modo se pueden incorporar los relatos y formatos crossmedia y transmedia a los procesos de enseñanza y aprendizaje de ELE. A partir de nuestra experiencia docente e investigadora, en este análisis argumentamos la necesidad de revisar los modelos pedagógicos en los que se basan las clases de contenido/ cultura en ELE para reconectar y motivar a nuestros alumnos prosumidores (que consumen, producen y mezclan textos multimodales y los comparten a través de diversas plataformas). Planteamos que las clases de cultura desde el prisma de una educación transmedia activan y potencian el sustrato crítico, emocional y colaborativo que subyace en los relatos y prácticas transmedia y, por lo tanto, ayuda a desarrollar la competencia intercultural. Desde esta perspectiva, abordamos la cuestión en primer lugar examinando diversos modelos pedagógicos que otorgan diferente importancia a la integración del aprendizaje de una lengua por contenidos en el marco de la educación de L2 en el Reino Unido. En el siguiente apartado, presentamos los proyectos centrados en la educación transmedia aplicados a la enseñanza de lenguas que se están realizando desde el Grupo de Investigación Film, Languages and Media in Education de Manchester Metropolitan University (FLAME). El último apartado incluye las conclusiones y futuras líneas de investigación.

\section{Modelos pedagógicos para la enseñanza de lenguas}

Como apunta Klee (2019: 492), el aprendizaje por contenidos "combina la enseñanza de un contenido académico particular con la adquisición de una lengua meta". Según Stryker y Leaver, las características fundamentales de este tipo de enseñanza incluyen las siguientes:

1) el núcleo de instrucción y su organización fundamental se derivan de la materia académica en vez de las formas o funciones lingüísticas; 2) los 
materiales de instrucción, incluyendo los textos escritos y los medios audiovisuales, son auténticos, es decir, diseñados para hablantes nativos de la lengua meta y no para aprendices de una segunda lengua (L2); y 3) el contenido y las actividades de aprendizaje corresponden a las necesidades lingüísticas, cognitivas y afectivas de los alumnos y por lo tanto, el plan curricular debe ser dinámico y flexible para poder acomodar a las diferentes exigencias de los estudiantes (citado por Klee, 2019: 492).

Entre los modelos que defienden el aprendizaje por contenidos se encuentra el Aprendizaje Integrado de Contenidos y Lenguas Extranjeras (AICLE) -en inglés Content and Language Integreated Learning (CLIL)- que ha ido ganando terreno como una metodología apropiada para integrar la lengua extranjera o segunda lengua como un modo de instrucción para otras asignaturas o materias curriculares. Como indican diversos expertos, en realidad este término incluye diferentes métodos, materiales y organización curricular que se adaptan a las necesidades educativas de diferentes países (Coyle 2007; Coyle, et al, 2010; Fortanet-Gómez, 2013; Boyler et al. 2020; Montaner-Villalba y Gimeno-Sanz, 2021). Coyle (1999) propone un marco conceptual que enfatiza la perspectiva holística de los diferentes aspectos de las pedagogías del AICLE y que se enfoca en la integración del aprendizaje (contenido y cognición), aprendizaje de la lengua (comunicación y culturas) y las experiencias interculturales. El marco de las cuatro Ces (contenido, comunicación, cognición y cultura) se basa en los siguientes principios: 1) la construcción del conocimiento por parte del aprendiz y el desarrollo de las competencias relevantes y apropiadas; 2 ) aprendizaje y pensamiento van de la mano y por lo tanto el contenido debe ser apropiado y accesible a los estudiantes; 3 ) los procesos cognitivos requieren que se evalúe la demanda lingüística a fin de facilitar el desarrollo de la misma; 4) el lenguaje necesita ser aprendido en contexto; 5) la interacción es fundamental para el aprendizaje, sea de forma escrita o hablada; 6 ) la interrelación entre culturas y lenguas es compleja y por lo tanto es importante que se tenga en cuenta a la hora de impulsar la comprensión intercultural y otras formas de ciudadanía global (Coyle, 2007).

La introducción de este tipo de metodología en la enseñanza en todos los niveles educativos tanto, tanto en ámbitos de aprendizaje formal como informal, se ha ido extendiendo paulatinamente. EI AICLE ha sido recomendado por diferentes instituciones europeas como una de las mejores formas de promover el aprendizaje pluricultural y plurilingüe. Sin embargo, el enfoque metodológico AICLE apenas está establecido en las escuelas del Reino Unido, salvo algunas notable excepciones. Desde el punto de vista pedagógico, la política educativa gubernamental británica a nivel de Secundaria ha propiciado un modelo que da mayor validez al estudio de las formas lingüísticas, dejando en un segundo plano el aspecto cultural. En noviembre de 2016, el Teaching Schools Council publicó un informe titulado Modern Foreign Languages Pedagogy Review. ${ }^{2}$ Este estudio, realizado por

\footnotetext{
${ }^{2}$ El Teaching Schools Council (TSC) busca mejorar y cambiar la educación en las escuelas inglesas (http://tscouncil.org.uk/).
} 
Ian Bauckham, presentaba las razones por las que el estudio de lenguas en Secundaria había ido mermando y señalaba con preocupación el marcado declive histórico en los $A$ Level de francés y alemán, un fenómeno que se había reflejado de forma similar en el estudio de estos idiomas en la universidad. El informe defendía una planificación curricular que daba preferencia a la enseñanza de vocabulario, la progresión gramatical y se ponía un mayor énfasis en la pronunciación y la traducción (Bauckham, 2016). En el año 2018 se crea el National Centre for Excellence for Language Pedagogy (NCELP), que cuenta con el apoyo del Departamento de Educación y está co-dirigido por la Universidad de York y el Cam Academy Trust. Su objetivo es implementar las recomendaciones del informe Modern Foreign Languages Pedagogy Review y, a través de la colaboración entre investigadores, profesores de lenguas y expertos en educación, elaborar materiales, recursos y ofrecer jornadas de formación. Por lo tanto, el NCELP propone una perspectiva para la enseñanza de lenguas en la que prima la atención dedicada a la gramática, la adquisición de vocabulario y la pronunciación. Siguiendo esta línea, la nueva propuesta curricular para Lenguas en Secundaria (GCSE) planteada por el Departamento de Educación en el 2021 continúa el mismo modelo desdeñando el estudio de temas culturales. $^{3}$

Diversas instituciones, como la Association for Language Learning (ALL) y el University Council of Modern Languages (UCML), vienen reclamando desde hace tiempo que el currículo de lenguas del Reino Unido se alinee a las tendencias pedagógicas actuales. ${ }^{4}$ La $A L L$ ha apoyado la adopción del AICLE. El proyecto FLAME, iniciado en 2012, tenía como objetivo la promoción de la enseñanza bilingüe. Estos objetivos han continuado con la colaboración de ALL con el proyecto ELAPSE ERASMUS + KA2 (2018-2020). ${ }^{5}$ ELAPSE busca diseminar la metodología CLIL/AICLE, incluida la denominada soft CLIL que se enfoca más en el aprendizaje de la lengua, entre los profesores de lenguas en la enseñanza de Primaria y Secundaria. Además, se ofrecen recursos y otros materiales en francés, alemán, español e inglés. ${ }^{6}$ Otra iniciativa en este ámbito es el proyecto Learning through Languages UK que está dirigido a la educación en colegios, pero que aspira a conectar desde este modelo el aprendizaje de lenguas

\footnotetext{
${ }^{3}$ Véase la siguiente entrada de blog que incluye lista de argumentos y contraargumentos enunciados por diversas instituciones: http://helenmyers.blogspot.com/2021/11/jointstatement-25th-november-2021-gcse.html

4 Establecido en 1993, El UCML presenta la opinión de docentes y profesionales especialistas en la enseñanza de lenguas moderna y publica regularmente informes sobre la situación de la enseñanza de lenguas en el Reino Unido (https://university-councilmodern-languages.org). La ALL es la asociación de profesores de lenguas de primaria y secundaria del Reino Unido, en la que participan también futuros docentes y profesores universitarios (https://www.all-languages.org.uk).

5 Más información sobre este proyecto se puede encontrar en el siguiente enlace: https://Ifee.net/erasmus-plus-elapse/.

${ }^{6}$ Véase el área dedicada a esta metodología en la página web de la ALL: https://www.alllanguages.org.uk/research-practice/clil-zone/.
} 
desde primaria hasta la universidad. ${ }^{7}$ Precisamente, una de las recomendaciones de la Comisión Europea (Cultura y Educación) desde el 2003 ha sido mejorar y facilitar el aprendizaje de lenguas a través de nuevas metodologías pedagógicas, como el Aprendizaje Integrado de Contenidos e Idiomas, especialmente a nivel universitario (Comisión Europea, 2003). Este llamamiento concuerda con el argumento que establecimos en la introducción sobre la importancia de romper con la falsa dicotomía de lengua y contenido. Constituye asimismo una manera de promover habilidades transversales: la resolución de problemas, el trabajo en equipo, el desarrollo de competencia intercultural y el análisis crítico, entre otras.

\section{Docencia e investigación sobre prácticas transmedia para la integración de lengua y contenido}

Venimos defendiendo desde hace tiempo el valor de trabajar con los medios de comunicación audiovisuales y explotarlos de una manera activa y efectiva en los programas educativos de ELE (lengua y contenido); una demanda que es ineludible en la actual era de interconectividad global dada la versatilidad que brinda la tecnología móvil y la mayor importancia de los contenidos audiovisuales y multimedia en todas las esferas de nuestra vida. Entre las funciones y aplicaciones pedagógicas de los medios audiovisuales cabe destacar su utilidad para desarrollar simultáneamente las competencias lingüísticas (inter)culturales y digitales y expandir el alfabetismo mediático (Herrero, 2018; Herrero, 2019b; Herrero, 2019c; Herrero y Vanderschelden, 2020). ${ }^{8}$

El papel estelar de las imágenes y las pantallas en la era actual se inscribe en un nuevo ecosistema de comunicaciones. En el siglo XXI estamos experimentado el vertiginoso desarrollo de las tecnologías de la información y la comunicación y la democratización de las nuevas herramientas de la Web 2.0. Estas nuevas tecnologías posibilitan la interactividad en ecosistemas digitales que construyen significado a través de la interacción de diferentes elementos (icónicos, verbales y sonoros) que conforman los textos multimodales. Todas estas transformaciones están modificando la forma en la que nos comunicamos y, por lo tanto, el modo en que realizamos nuestras actividades laborales y personales (ocio, cultura, etc.). En la esfera educativa, estos cambios implican nuevas literacidades que esencialmente son más participativas, colaborativas y distribuidas (Lankshear y Knobel 2008). La actual cultura participativa facilita y promueve nuevas prácticas creativas basadas en la creación de artefactos digitales en diferentes formatos (Jenkins, 2008). Esta cultura, caracterizada por "relatively low barriers to artistic expression and civic engagement, strong support for creating and sharing one's creations", facilita el aprendizaje informal entre

\footnotetext{
${ }^{7}$ Los objetivos de este proyecto y las actividades y recursos que ofrecen se encuentran disponibles en el siguiente enlace: https://learningthroughlanguages.org.

${ }^{8}$ Este modelo ha sido promovido por la asociación Film in Language Teaching Association (FILTA) (Herrero, 2016).
} 
pares ya que se valora y se anima a que compartan estos productos (Jenkins et al., 2006: 3). El proyecto de investigación dirigido por Jenkins para la Fundación McArthur identificó una serie de prácticas y habilidades propiciadas por este nuevo sistema mediático que genera conexiones afectivas e identitarias. Entre las nuevas habilidades de los prosumidores se incluyen las siguientes: apropiación (similar al remix), capacidad crítica, inteligencia colectiva, juego, multitarea, navegación transmedia, negociación, networking, pensamiento distribuido, representación, simulación y visualización (Jeninks et al., 2006).

No cabe duda de que la diversificación y accesibilidad a diversos tipos de pantallas ha multiplicado la posibilidad de consumir y producir relatos en los que se manifiestan identidades y estéticas diversas. La convergencia mediática abre una puerta al proceso expansivo de las "narrativas transmedia" (en inglés transmedia storytelling), "historias contadas a lo largo de varios medios (...) [que ] tienden a fluir entre una diversidad amplia de medios y plataformas de comunicación" (Scolari, 2013:53). ${ }^{9}$ De ahí que la navegación transmedia, definida como "the ability to deal with the flow of stories and information across multiple modalities" (Jenkins et al., 2006: 46), resulte una de las más relevantes para el mundo educativo. ${ }^{10}$ No obstante, la revisión de los trabajos de investigación en el apartado dedicado a transmedia y educación revela que hay pocos estudios relacionados con la aplicación de las narrativas transmedia a la enseñanza y el aprendizaje de lenguas extranjeras, particularmente a nivel terciario (González Martínez et al., 2019; Andrade Velásquez y Fonseca Mora, 2021).

En el marco del trabajo realizado por este Grupo de Investigación Film, Languages and Media in Education de Manchester Metropolitan University (FLAME), una de las áreas de investigación ha sido explorar la posible aplicación de las narrativas transmedia audiovisuales a la enseñanza de segundas lenguas. Además, el hecho de que los textos culturales durante ese proceso de circulación se transforman y re-contextualizan constituye un hecho particularmente útil en las clases de cultura de L2 para explotar como recurso, estudiar como parte del contexto de otros textos y/o explorar en profundidad como materia de estudio. Apoyado por el Arts and Humanities Research Council bajo el programa de investigación Open World Research Initiative (OWRI), ${ }^{11}$ el proyecto New Approaches to Transmedia and Language Pedagogy (2018-2021) tenía, entre otros, los siguientes objetivos: 1) investigar como los proyectos transmedia podían complementar los modelos tradicionales de enseñanza y aprendizaje de L2; y 2) experimentar con diversos escenarios que apoyaran el desarrollo de prácticas educativas

\footnotetext{
9 Véase también Jenkins, 2003; 2006; 2010; 2013; Freeman y Gambaro, 2009; Scolari, 2018.

10 Muestra de su importancia en la esfera de la educación es el Proyecto Transmedia Literacy, financiado por el programa Horizon 2020 de la Comisión Europea. Su objetivo era identificar las habilidades transmedia empleadas en entornos de aprendizaje informal y examinar como se podían aplicar al sistema educativo formal (Scolari, 2018: 9).

11 Para obtener más información sobre el proyecto Cross-Language Dynamic, véase la página del mismo: http://projects.alc.manchester.ac.uk/cross-language-dynamics/aboutthe-project/.
} 
innovadoras centradas en los estudiantes por medios de estrategias colaborativas encaminadas a desarrollar las competencias y habilidades que se requieren para ser un ciudadano competente y plenamente funcional (Herrero, 2019a; Transmedia in Education). ${ }^{12}$

Como señala Pratten (2019), la educación transmedia implica enfocarse en los estudiantes: "building a learning environment around the learner" ("a training audience"). Además, hay que considerar el perfil del aprendiz y su comportamiento fuera del aula, "trying to fit in with their existing behaviour". Se debe ofrecer un aprendizaje holístico que vaya a la par con el estilo de vida del alumno. Es decir, es fundamental hacer que el aprendizaje sea participativo y una experiencia motivadora que sea relevante, contextuada y con una meta. Las implicaciones de esta metodología van más allá del aprendizaje realizado durante los estudios universitarios, ya que lo que hacen es comenzar "a longer journey of problem-solving" (Pratten, 2019: online). Dada la importancia en la educación transmedia de un aprendizaje eminentemente activo y centrado en los estudiantes, uno de los primeros pasos del proyecto New Approaches to Transmedia and Language Pedagogy fue identificar las habilidades que nuestros estudiantes desarrollan en el marco de la cultura participativa en la que están inmersos. Esta información permitiría planificar modelos curriculares que respondieran a esos cambios indicados y a los perfiles de los estudiantes, tanto en las clases de contenidos como en los modelos de docencia y evaluación. Al mismo tiempo, se hizo una revisión de diversos modelos pedagógico que se ajustaban a estos nuevos parámetros: la pedagogía de la mirada (Dussell y Gutiérrez, 2006); la pedagogía de la participación (Piscitelli, 2009); la educación emocional a través de las pantallas (Ferrés, 2008; 2014); educación y transmediaciones (OrozcoGómez, 2010); el aprendizaje invisible (Cobo-Romani y Moravec, 2010); la educación de la interactividad (Aparici y Silva, 2012); el aprendizaje en conexión (en inglés connected learning) (Ito et al., 2013); y la pedagogía transmedia (Scolari, 2014).

Asimismo, se evaluaron las experiencias didácticas de anteriores trabajos de investigación en las que los aprendices debían ser capaces de visualizar historias y ampliar sus habilidades comunicativas y otras destrezas relacionadas con el uso de medios (competencia digital, crítica, creativa...). Basados en las heterogéneas prácticas de comunicación multimodal, estos proyectos interdisciplinares incluían tareas reales representativas de las que se llevan a cabo fuera del aula (Herrero et al., 2017; Herrero y Escobar, 2018). En particular, el proyecto "Film and Creative Engagement", una colaboración entre Manchester Metropolitan University el Instituto Tecnológico de Estudios Superiores de Monterrey (ITESM) de México, permitió crear contextos para la comunicación interactiva basada en tareas

12 Las competencias del siglo XXI que se ajustan a este nuevo paradigma incluyen adaptabilidad, creatividad, pensamiento crítico, curiosidad, resolución de problemas, comunicación, colaboración, iniciativa, liderazgo, persistencia, resiliencia, iniciativa y la competencia intercultural. 
en las que los estudiantes desarrollaron diversas destrezas por medio de análisis fílmico (como el conocimiento cultural e intercultural), y la accesibilidad audiovisual (subtítulos para sordos y para personas con problemas auditivos y audio-descripción). Este proyecto ayudó a que los alumnos mejorasen sus conocimientos lingüísticos, el trabajo colaborativo, el análisis crítico, la innovación y la creatividad, el manejo de la información y las tecnologías de la comunicación; asimismo, promovió la colaboración internacional en entornos virtuales (Herrero et al. 2020).

En las unidades de "contenido" ofrecidas como parte del programa de español de Manchester Metropolitan University, se realizó un rediseño curricular acorde a los modelos pedagógicos enunciados y a las directrices de una educación transmedia. Por ejemplo, para la unidad Power and Culture in Spain, se seleccionaron contenidos que ofreciera aspectos culturales relevantes y que al mismo tiempo permitieran desarrollar las destrezas lingüísticas e interculturales. En la evaluación se optó por aprovechar plenamente las posibilidades que ofrecen los nuevos géneros y prácticas digitales que requieren el dominio de la escritura analógica y un buen conocimiento pragmático del contexto cultural y social en el que se enmarcan la creación e interpretación de cada texto digital (entradas de blogs, podcasts y videos (video-essays) que se complementaban con presentaciones orales y/o ensayos. A través del análisis de diversos tipos textos y ejemplos de actividades trabajados en la clase, se encamina a los alumnos a comunicarse en y para el entorno digital que requiere conocer y practicar las características propias de la escritura digital (multimodalidad, multimedia, hipertextualidad, colaborativa, etc.) y otras formas de comunicación. Como resultado de la nueva cultura participativa, la colaboración entre los alumnos en la composición digital para crear entornos de trabajo en los que comunicarse almacenar y editar de forma colaborativa documentos de formar virtual y/o compartir archivos constituye un componente fundamental. Esta forma de trabajo colaborativo se introdujo en unidades como Contextualising Language Learning donde una de las tareas de evaluación incluye la creación de un póster o una infografía (una representación gráfica en forma de cartel que, a través de la combinación de textos, elementos gráficos y datos, comunica de forma visual, sintética y clara una gran cantidad de información) para mostrar el resultado del trabajo de investigación del grupo. En la selección de los textos primarios y secundarios para la unidad Shifting Borders in Latin America and Spain se consideró fundamentalmente la dimensión pluricultural y plurilingüe. Por ello se dedicó especial atención a al criterio de selección de material fílmico y literario, atendiendo a criterios pedagógicos, didácticos, temáticos, lingüísticos y a la dimensión intercultural de los mismos (encuentros de culturas y discursos de poder) que permitiera trabajar taxonomías cognitivas y afectivas (sensibilización, concienciación, relativización, comparaciones, etc.). Finalmente, la asignatura Transnational and Transmedia Practices in Latin America and Spain, que aborda producciones transnacionales y ejemplos de narrativa transmedia de la 
cultura contemporánea, se centra directamente en el fenómeno de la convergencia cultural y se estudian las tendencias en esta área a partir de series de televisión, películas, obras literarias y videojuegos. Como parte del portfolio para esta unidad, además de una presentación oral en español, los estudiantes han crear un blog en el que comparten las tareas requeridas para la evaluación de la unidad.

Por otro lado, se anima a los estudiantes a que participen en un proyecto extracurricular directamente ligado a la experiencia de las narrativas transmedia iniciado en el 2019. Transmedia Practices (Open Educational Resources for Students by Students) tiene como objetivo alentar a que los estudiantes se familiaricen con conceptos como recursos educativos abiertos, las licencias creativas (Creative Common Licences) y a que creen y compartan recursos educativos abiertos (Herrero, en prensa). Se basa en la noción de reusar los trabajos creados como parte de la evaluación de las unidades que han estudiado. Los estudiantes comparten, adaptan o expanden sus trabajos en diferentes formatos con una licencia CC, lo que contribuye a mejorar el conocimiento en diversas áreas y prácticas de futuros estudiantes. Se trata de un establecer un puente entre las estrategias de aprendizaje formal e informal que permita que los alumnos actúen como prosumidores y desarrollen diversas habilidades como el remix, la capacidad crítica, la inteligencia colectiva, la navegación transmedia, negociación, y la visualización (Jeninks et al, 2006). Junto con podcasts, la mayoría de los artefactos son narraciones digitales en formato de video. Todos los materiales creados como parte de este proyecto (videos y podcasts) se encuentran disponibles en el siguiente enlace: https://bit.ly/373xAOV.

\section{Conclusiones}

Las narrativas transmedia están revolucionando la ecología mediática y las formas culturales al incorporar historias contadas por diversos medios (película, serie web, novela gráfica o videojuego) dentro de un único universo narrativo. En cierto sentido, las narrativas transmedia pueden ser vistas como una metáfora de la integración de lengua y contenido en la enseñanza de L2: historias contadas a través de diversos medios que se expanden, se complementan y que en esa interacción enriquecen el proceso de aprendizaje y contribuyen a aumentar la motivación de los aprendices. Por ello creemos que es posible desde la educación transmedia complementar y expandir la perspectiva curricular mediante una concepción dinámica, creativa y participativa de las clases. Se trata de utilizar todos los recursos disponibles en la clase de cultura que apoyen tanto la adquisición de un determinado contenido académico como la de la lengua. A nuestro juicio, el reto no es solo ajustar los objetivos a los niveles y características de los alumnos, sino apelar a la emoción y la cognición y sintonizar con las formas de comunicación actuales. Hemos argumentado que, a través de un aprendizaje eminentemente activo y centrado en los 
estudiantes y por medio de una modelo de educación transmedia, se incrementa el repertorio de géneros y las estrategias de aprendizaje. Este tipo de experiencia educativa permite que los estudiantes desarrollen no solo las competencias lingüísticas, (inter)cultural, crítica, audiovisual/fílmica y digital, sino que también aprendan nuevas estrategias de aprendizaje, en un entorno formal e informal, en el que los estudiantes actúan como mentores.

También cabe destacar el valor de este tipo de prácticas transmedia por sus características fundamentales (autenticidad y flexibilidad) para no solo confrontar los desafíos que ha traído la era post-Covid 19, sino también aprovechar las oportunidades que nos brinda. Sin duda estamos ante una nueva etapa en el mundo de la educación donde es más beneficioso generar un conocimiento que conecte con situaciones, necesidades y aplicaciones de la vida real. Por ello, una de las finalidades de este tipo de proyectos en los que se integra la educación transmedia es desarrollar el pensamiento crítico a partir de contenidos curriculares que derivan de temas o formas comunicativas vinculadas a la realidad de los aprendices. Es fundamental hacer que los estudiantes sean co-creadores de recursos y materiales y que sus artefactos contribuyan a crear una comunidad de práctica.

Lo cierto es que nos encontramos en estos momentos ante el comienzo de una etapa donde convergen numerosos modelos pedagógicos. Lo pertinente ahora es ampliar las experiencias en diversos países, con diferentes lenguas y grupos a fin de recoger y analizar los resultados para poder medir de forma empírica el potencial didáctico de la narrativa transmedia a la hora de apoyar el aprendizaje integrado de contenidos y lengua.

En definitiva, se puede y se debe promover la educación trasmedia por muchas razones, pero siempre poniendo el foco en la exigencia académica y la calidad de la experiencia de los estudiantes. Además, dada la creciente importancia de la participación de los aprendices como cocreadores de un currículo que enriquezca sus conocimientos de la materia y la lengua, la narrativa transmedia representa un recurso polivalente, motivador y accesible (gracias a las nuevas plataformas de streaming) que creemos puede enriquecer la formación de los alumnos y complementar la labor docente del profesor.

\section{BiBLIOGRAFÍA}

Andrade-Velásquez, M. R. y FonseCA-Mora, M. D. (2021): "Las narrativas transmedia en el aprendizaje de lenguas extranjeras". Revista Mediterránea de Comunicación, 12 (2), pp. 159-175.

APARICI, R. y SILVA, M. (2012): "Pedagogía de la interactividad”. Comunicar, 19 (38), pp. 51-58.

BRITISH ACADEMY (2016): Born Global. http://www.britac.ac.uk/born-global

BRITISH ACADEMY (2019): Languages in the UK/A call for action from om the four UK-wide National Academies: The British Academy; with the 
Academy of Medical Sciences; the Royal Academy of Engineering; and the Royal Society. https://university-council-modernlanguages.org/wp-content/uploads/2020/05/Languages-UK-2019academies-statement.pdf

BOWLER, M. (2020): A language crisis? High Education Policy Institute (HEPI) report $123 . \quad$ https://www.hepi.ac.uk/wpcontent/uploads/2020/01/HEPI A-Languages-Crisis Report-123FINAL.pdf

Boyer, K., Coyle, D., Cross, R. y Chambers, G. N. eds., (2020): Curriculum integrated language teaching: CLIL in practice. Cambridge University Press.

BROADY, E. (2020): "Language learning in the UK - taking stock", The Language Learning Journal. 48 (5), pp. 501-507.

BAUCKLAN, I. (2016): Modern Foreign Languages Pedagogy Review. https://tscouncil.org.uk/wp-content/uploads/2016/12/MFLPedagogy-Review-Report-2.pdf

Сово, C. у Moravec, J. W. (2011): Aprendizaje invisible: Hacia una nueva ecología de la educación. Col-lecció Transmedia XXI. Barcelona, Laboratori de Mitjans Interactius / Publicacions i Edicions de la Universitat de Barcelona.

ColleN, I. (2021): Languages Trends 2021. Language Teaching in Primary and Secondary Schools in England. British Council. https://www.britishcouncil.org/research-policy-insight/researchreports/language-trends-2021

COMISIÓN EUROPEA (2003): "Promover el aprendizaje de idiomas y la diversidad lingüística: Un Plan de acción 2004-2006". https://eurlex.europa.eu/LexUriServ/LexUriServ.do?uri=OJ:C:2004:073:0033:0 037:ES:PDF

COMISIÓN EUROPEA (2019): "Foreign language skills statistics". https://ec.europa.eu/eurostat/statisticsexplained/index.php/Foreign language skills statistics.

COYLE, D. (1999): "Theory and planning for effective classrooms: Supporting students in content and language integrated learning contexts". Learning through a foreign language, pp.46-62.

COYLE, D. (2007): "Content and Language Integrated Learning: Towards a Connected Research Agenda for CLIL Pedagogies". International Journal of Bilingual Education and Bilingualism, 10 (5), pp. 543-562.

COYLE, D., HOOD, P. y MARSH, D. (2010): Content and Language Integrated Learning. Nueva York, Cambridge University Press.

DENECHI, S., LOFT, P. y LONG, R (2020): Language Teaching in Schools (England, UK). [online] UK Parliament - House of Commons Library. House of Commons Library: House of Commons. https://commonslibrary.parliament.uk/

DoBson, A. (2020): "Context is everything: reflections on CLIL in the UK", The Language Learning Journal, 48 (5), pp.508-518, 
Dussel, I., y GutiérRez, D. (2006): Educar la mirada. Buenos Aires, Ediciones Manantial.

FERRÉS, J. (2008): La educación como industria de deseo, Barcelona, Gedisa.

FERRÉS, J. (2014): Las pantallas y el cerebro emocional, Barcelona, Gedisa.

FILTA (Film in Language Teaching Association): www.filta.org.uk

FLAME (Film, Languages and Media in Education)- Manchester Metropolitan University: (https://www2.mmu.ac.uk/languages/flame/

TRANSMEDIA IN EDUCATION. www.transmediaineducation.com

ForTANET-GómEZ, I. (2013): CLIL in Higher Education.Towards a Multilingual Language policy. Londres, Reino Unido, Multilingual Matters.

GIEVE, S. y CUNICO, S. (2012): "Language and content in the modern foreign languages degree: A students' perspective". The Language Learning Journal, 40 (3), pp. 273-291

Gorrara, C., Jenkins, L., Jepson, E. y LleWelyn Machin, T. (2020): “Multilingual perspectives: preparing for language learning in the new curriculum for Wales". The Curriculum Journal, 31 (2), pp. 244-257.

Herrero, C. (en prensa). "Transmedia Practices in Higher Education: Open Educational Resources for Students by Students". J. Bobkina et al. (eds.), Visual Literacy and Digital Communication. The Role of Media in New Teaching Practices. Madrid: Comares.

Herrero, C. (2016): "Film in Language Teaching Association (FILTA): a Multilingual Community of Practice". English Language Teaching Journal 70 (2), pp. 190-199.

Herrero, C. (2018): "El cine y otras manifestaciones culturales en ELE". M. Martínez-Atienza de Dios y A. Zamorano Aguilar (eds.), Iniciación a la metodología de la enseñanza de ELE. IV. Literatura, cine y otras manifestaciones literarias. Madrid: enCLAVEELE, pp. 65-82.

Herrero, C. (2019a): "Escritura digital: estrategias de enseñanza-aprendizaje en entornos digitales". AA.VV, Lectoescritura digital. Madrid: Ministerio de Educación, Cultura y Deporte, pp. 123-132.

Herrero, C. (2019b): "From new literacies to transmedia literacies: New Approaches to Transmedia and Languages Pedagogy project". N. Becerra, R. Biasini, H. Magedera-Hofhansl, y A. Reimão (eds.), Innovative language teaching and learning at university: a look at new trends, Research-publishing.net., pp. 19-26.

Herrero, C. (2019c): "Medios audiovisuales (Audiovisual media)". MuñozBasols, E. Gironzetti y M. Lacorte (eds.), The Routledge Handbook of Spanish Language Teaching: metodologías, contextos y recursos para la enseñanza del español L2. London y New York: Routledge, pp. 565582.

Herrero, C. y Escobar, M. (2018): “A Pedagogical Model for Integrating Film Education and Audio Description in Foreign Language Acquisition". Translation and Translanguaging in Multilingual Contexts, 4 (1), pp. 30-54. 
Herrero, C., et al. (2020): "The Film and Creative Engagement Project: Audiovisual Accessibility and Telecollaboration". Research in Education and Learning Innovation Archives, 24, pp. 89-104.

Herrero, C., SÁnchez-RequenA, A. y Escobar, M. (2017): “Una propuesta triple: Análisis fílmico, traducción audiovisual y enseñanza de lenguas extranjeras". inTRAlinea. Online Translation Journal. Special issue on Building Bridges between Film Studies and Translation Studies. http://www.intralinea.org/specials/article/una propuesta triple a nalisis filmico traduccion audiovisual y ensenanza

Herrero, C. y Vanderschelden, I. (eds.) (2019): Using Film and Media in the Language Classroom: Reflections on Research-led Teaching. Bristol: Multilingual Matters.

ITO, M. et al. (2013): Connected learning: An Agenda for Research and Design. Digital Media and Learning Research Hub.

JeNKINS, H. (2008): Convergence culture: La cultura de la convergencia de los medios. Barcelona: Paidós.

JenkINS, H. et al. (2006): Confronting the Challenges of Participatory Culture: Media Education for the $21^{\text {st }}$ Century. The McArthur Foundation

KLEE, C. A. (2018): “Aprendizaje por contenidos e inmersión lingüística (Content-based learning and language immersion)". Muñoz-Basols, E. Gironzetti y M. Lacorte (eds.), The Routledge Handbook of Spanish Language Teaching: metodologías, contextos y recursos para la enseñanza del español L2. London y New York: Routledge, pp. 491504.

LANKSHEAR, C. y KNOBEL, M. (2008): Nuevos alfabetismos. (Su práctica cotidiana y el aprendizaje en el aula). Madrid: Editorial Morata.

Kelly, M. (ed.) (2018): Languages after Brexit. Springer International Publishing.

Montaner-Villalba, S. y Gimeno-SAnZ, A. M., (2021): "Research in TechnologyEnhanced Content and Language Integrated Learning (CLIL)". VERBEIA. Revista de Estudios Filológicos. Journal of English and Spanish Studies, 5, pp. 6-9.

Orozco GómEZ, G. (2011): "Debemos formar audiencias críticas y activas", https://youtu.be/qwsGijMsHo

PARKS, E. (2020a). Developing Critical Cultural Awareness in Modern Languages. A comparative study of Higher Education in North America and the United Kingdom, New York, Routledge.

PARKS, E. (2020b): “The separation between language and content in Modern Language degrees: implications for students' development of critical cultural awareness and criticality", Language and Intercultural Communication, 20 (1), pp. 22-36,

PISCITELLI, A. (2009): Nativos digitales, dieta cognitiva, inteligencias colectivas y arquitecturas de participación. Montevideo: Editorial Santillana.

Pratten, R. (2019): "Transmedia in Education: an interview with Robert Pratten". https://mmutube.mmu.ac.uk/media/Transmedia+and+Education- 
+Interview+with+Robert+Pratten.+Interview+by+Carmen+Herrero/ 1 e7wkfs5b

SCOLARI, C. (2013): Narrativas transmedia: cuando todos los medios cuentan. Barcelona: Deusto.

SCOLARI, C. (2018): Transmedia literacy in the new media ecology: white paper = Alfabetismo transmedia en la nueva ecología de los medios: libro blanco. Barcelona: Universitat Pompeu Fabra, Departament de Comunicació.

WILKINSON, R. (2018): “Content and language integration at universities? Collaborative reflections", International Journal of Bilingual Education and Bilingualism, 21 (5), pp. 607-615.

Worton, M., (2009): Review of Modern Foreign Languages Provision In Higher In Education England. https://discovery.ucl.ac.uk/id/eprint/329251/2/hereviewworton.pdf

ZAPPA-Hollman, S. (2018): "Collaborations between language and content university instructors: Factors and indicators of positive partnerships". International Journal of Bilingual Education and Bilingualism, 21 (5), pp. 591-606. 